\title{
Predictors of Cardiovascular Autonomic Neuropathy in Diabetic Patients: A Cross- Sectional Study from Basrah
}

\author{
Abbas Ali Mansour ${ }^{1}$ and Akream Hussain Odea ${ }^{2}$ \\ ${ }^{1}$ Department of Medicine, Basrah College of Medicine, Basrah, Iraq \\ ${ }^{2}$ Department of Medicine, Al-Faiha Hospital, Basrah, Iraq
}

Correspondence should be addressed to: Abbas Ali Mansour; aambaam@yahoo.com

Received 21 March 2013; Accepted 7 May 2013; Published 18 July 2013

Academic Editor: Serap Baspinar Demir

Copyright (C) 2013 Abbas Ali Mansour and Akream Hussain Odea. Distributed under Creative Commons CC-BY 3.0

\begin{abstract}
Background: Diabetes mellitus is the most common cause of autonomic neuropathy. The aims of this study were to determine the prevalence of cardiovascular autonomic neuropathy (CAN) in diabetic patients in Basrah by conducting cardiac autonomic function tests as well as to know the predictors of the CAN in diabetic patients.

Methods: The study was conducted at the Al-Faiha Diabetes Endocrine and Metabolism Center (FDEMC) in Basrah for the period from January to December 2011. Sixty eight patients were selected to participate in this study. The tests for autonomic cardiovascular function were performed as bedside procedures.

Results: The CAN was seen in $42.6 \%$ of the selected diabetic patients. The sequence of abnormal tests in this study was as follows: beat-to-beat heart rate variation (E/I), heart rate response to Valsalva maneuver, diastolic blood pressure response to isometric exercise, and then heart rate response to standing. Possible predictors of CAN were gastroparesis, nocturnal diarrhea, areas of symmetrical anhydrosis, gustatory sweating, dry skin, erectile dysfunction for men, palpitation, exercise intolerance and insulin use, but not the duration of diabetes.
\end{abstract}

Conclusion: Various variables can predict the CAN, which affects more than $40 \%$ of diabetic patients in Basrah.

Keywords: Diabetes,cardiac autonomic neuropathy, predictors.

Cite this Article as: Abbas Ali Mansour and Akream Hussain Odea (2013), "Predictors of Cardiovascular Autonomic Neuropathy in Diabetic Patients: A Cross-Sectional Study from Basrah," Research in Endocrinology, Vol. 2013 (2013), Article ID 301170, DOI: 10.5171/2013.301170 


\section{Introduction}

Diabetes mellitus is the most common cause of autonomic neuropathy. And neuropathy is the most common complication of diabetes mellitus and may have both somatic and autonomic features. ${ }^{1}$ Autonomic decline includes (in order) peripheral autonomic neuropathy (PAN), then diabetic autonomic neuropathy (DAN), and finally cardiovascular autonomic neuropathy (CAN). ${ }^{2,3}$

There are three stages to the CAN. Early stage: abnormality of heart rate response during deep breathing alone. Intermediate stage: an abnormality of Valsalva response. Severe stage: the presence of postural hypotension. ${ }^{4}$

Clinical symptoms of autonomic neuropathy generally do not occur until long after the onset of diabetes. ${ }^{3,5}$ Although symptoms suggestive of autonomic dysfunction may be common, they may frequently be due to other causes rather than to the true autonomic neuropathy. Subclinical autonomic dysfunction can, however, occur within a year after diagnosis among type 2 diabetes patients and within two years among type 1 diabetes patients. ${ }^{6}$ Because of its association with a variety of adverse outcomes including cardiovascular deaths, the CAN is the most clinically important and well-studied form of the DAN.

The number of bedside tests used to diagnosed patients with the CAN are different from one study to another ranging from one to six. ${ }^{3}$ If more strict criteria were used (i.e., abnormalities present in at least three of six autonomic function tests), the prevalence of CAN was $16.8 \%$ for individuals with type 1 diabetes and $22.1 \%$ for individuals with type 2 diabetes. $^{3}$

Results from earlier research suggest that using a battery from cardiovascular tests (some indicating parasympathetic involvement and others indicating possible sympathetic involvement) would make it possible to follow the progression of autonomic function over time. ${ }^{7}$
The three tests recommended were heart rate response to 1) deep breathing, 2) standing, and 3) the Valsalva maneuver. Two tests of blood pressure control were also recommended: blood pressure response to 1 ) standing or passive tilting and 2) sustained handgrip. These tests were judged suitable for both routine screening and monitoring of the progress of autonomic neuropathy. ${ }^{4}$

In 1992, a consensus development conference on standardized measures in diabetic neuropathy review the state-of-theart diabetic neuropathy measures used in epidemiological and clinical studies including cross-sectional, longitudinal, and therapeutic trials. The panel revised its recommendation to include three tests for the longitudinal testing of the CAN: 1 ) heart rate response during deep breathing, 2) Valsalva maneuver, and 3) postural blood pressure testing. ${ }^{8}$

Aims of this study was to determine the prevalence of CAN in diabetic patients in Basrah by conducting cardiac autonomic function tests and to detect the predictors of the CAN in diabetic patients.

\section{Methods}

The study was conducted at the Al-Faiha Diabetes Endocrine and Metabolism Center (FDEMC) in Basrah for the period from January to December 2011. Sixty eight patients were selected to participate in this study. Written informed consent was obtained from each patient. Patients were selected irrespective of the duration of disease and therapeutic status. All patients were examined by a single investigator. Diabetes mellitus was defined as plasma glucose level being $200 \mathrm{mg} / \mathrm{dl}$ or higher independent of fasting time, fasting plasma glucose of $\geq 126 \mathrm{mg} / \mathrm{dl}$ on two occasions or, use of anti-diabetes agents or a combination of these. Current smoker was defined as any person who smoked cigarettes regardless of the number in the preceding three months .Hypertension was defined as systolic blood pressure being $140 \mathrm{mmHg}$ or higher, 
diastolic blood pressure being $90 \mathrm{mmHg}$ or higher, use of antihypertensive agents or a combination of these. Blood pressure was measured in a seated position after 5 minutes at rest, three times. The average of the second and third readings was defined as the subject's blood pressure. Diagnosis of peripheral neuropathy was based on quantitative assessment of symptoms and physical finding. ${ }^{9}$ Body mass index (BMI, $\mathrm{kg} / \mathrm{m} 2$ ) was computed from height and weight measurements.Exclusion criteria included those with poor functional capacity, amputation of any of the lower limbs, acute ischemic heart disease with admission to contrary care unit in the preceding month and cerbrovasuclar disease interfering with walking, standing or speech.

Clinical symptoms of autonomic neuropathy were assessed on the basis of presence or absence of various symptoms, such as nausea, vomiting, nocturnal diarrhea, constipation, early satiety, stool incontinence, gustatory sweating, bloating, fullness, dry skin, erectile dysfunction, urine retention, sterility or infertility, palpitation, syncope, or dizziness. The definitions of above symptoms were based on recommendation of American diabetic association $(\mathrm{ADA}){ }^{3}$

The tests for autonomic cardiovascular function performed as side procedures are described below . The tests were carried out in the morning. The participants arrived at 9 a.m, in the fasting state. Examination started with the patient resting in lying position for 5 minutes. A 12-lead ECG was performed with a standard device. The whole examination lasts 15-20 minutes per patient and no time for preparation is needed. The autonomic nervous system was examined using a battery from the five function tests.

\section{Cardiovascular Autonomic Function Tests}

1. Beat-to-beat heart rate variation (E/I) ratio (With the patient at rest and supine (no overnight coffee or hypoglycemic episodes), breathing 6 breaths/min, heart rate monitored by ECG , a difference in heart rate of $>15$ beats $/ \mathrm{min}$ is normal and $<10$ beats/min is abnormal, R-R expiration / R-R inspiration(E/I) $>1.17$ is abnormal ). (Parasympathetic activity).

2. Heart rate response to Valsalva maneuver $\{($ The subject forcibly exhales into the mouthpiece of a manometer to $40 \mathrm{mmHg}$ for $15 \mathrm{~s}$ during ECG monitoring. Healthy subjects develop tachycardia and peripheral vasoconstriction during strain and an overshoot bradycardia and rise in blood pressure with release. The ratio of longest R-R shortest R-R should be > 1.2)\}. (Parasympathetic activity)

3. Heart rate response to standing $\{(30: 15$ ratio) (During continuous ECG monitoring, the R-R interval is measured at beats 15 and 30 after standing. Normally, a tachycardia is followed by reflex bradycardia. The $30: 15$ ratio is normally $>$ 1.03)\}. (Parasympathetic activity)

4. Systolic blood pressure response to standing (systolic blood pressure is measured in the supine subject. The patient stands and the systolic blood pressure is measured after $2 \mathrm{~min}$. Normal response is a fall of $<10 \mathrm{mmHg}$, borderline is a fall of $10-29 \mathrm{mmHg}$, and abnormal is a fall of $>30 \mathrm{mmHg}$ with symptoms). (Sympathetic activity)

5. Diastolic blood pressure response to isometric exercise (handgrip) $\{($ The subject squeezes a handgrip dynamometer to establish a maximum. Grip is then squeezed at $30 \%$ maximum for $5 \mathrm{~min}$. The normal response for diastolic blood pressure is a rise of $>16$ mmHg in the other arm)\}. (Sympathetic activity)

If two of the aforementioned test results were abnormal, the presence of cardiovascular autonomic neuropathy was assumed.10 


\section{Biochemical Studies}

Fasting serum samples were analyzed in the same local laboratory. Each subject's blood was sampled: $10 \mathrm{~mL}$ was drawn into a tube after the subject had fasted for at least 10 hours. Serum total cholesterol, high density lipoprotein cholesterol (HDL-C), and triglyceride (TG) were measured by the enzymatic method. Low density lipoprotein cholesterol (LDL-C) concentrations were calculated using the Friedewald formula. $\mathrm{Hb}$ A1c was measured using Bio-Rad D10 analyzer (HPLC cation-exchange chromatography).

An ethical approval for this study was obtained from the Basrah directorate of health.

\section{Statistics}

The group data are presented as mean \pm standard deviation unless otherwise specified .A $\mathrm{p}$ value <0.05was considered significant. All analyses were performed using SPSS software version 18.0(SPSS, Chicago, IL, USA). Univariate analysis was performed to determine each variable's association with autonomic neuropathy. In the sixty eight patients, univariate analysis was performed for each of the factors with the presence or absence of autonomic neuropathy, then the presence of autonomic neuropathy used as dependent variable. Variables found associated with autonomic neuropathy in univariate analyses were then entered into a multivariable model using logistic regression to determine the strength of each variable in association with autonomic neuropathy.

\section{Results}

Autonomic neuropathy was presents in twenty nine patients (42.6\%) as shown in table one. Abnormalities of two tests were seen in thirteen patients $(19.1 \%)$, three tests in six $(8.8 \%)$, four tests in nine $(13.2 \%)$ and five tests in one $(1.5 \%)$.

Autonomic test results are summarized in table 2. Parasympathetic function: abnormal beat-to-beat heart rate variation $(\mathrm{E} / \mathrm{I})$ ratio are present in $61.8 \%$; abnormal heart rate response to Valsalva maneuver in $33.8 \%$; and abnormal heart rate response to standing in $23.5 \%$. Sympathetic function: abnormal diastolic blood pressure response to isometric exercise are present in $25 \%$, and orthostatic hypotension (defined as systolic blood pressure fall of $>30 \mathrm{mmHg}$ within 2 minutes of standing) in $5.1 \%$.

\section{Table-1- Autonomic Tests Abnormalities ( $=68$ )}

\begin{tabular}{|l|l|}
\hline Tests abnormalities & $\mathrm{N}(\%)$ \\
\hline 0 test & $22(32.3)$ \\
\hline 1 test & $17(25)$ \\
\hline 2 test & $13(19.1)$ \\
\hline 3 tests & $6(8.8)$ \\
\hline 4 tests & $9(13.2)$ \\
\hline 5 test & $1(1.5)$ \\
\hline
\end{tabular}


Table-2- Distribution of Patients according to the Autonomic Neuropathy Abnormality $(\mathrm{N}=68)$

\begin{tabular}{|l|l|l|}
\hline \multicolumn{2}{|l|}{ Normal N (\%) } & Abnormal N (\%) \\
\hline Parasympathetic neuropathy & $26(38.2)$ & $42(61.8)$ \\
\hline Beat-to-beat heart rate variation( E/I) & $45(66.2)$ & $23(33.8)$ \\
\hline Heart rate response to Valsalva maneuver & $52(76.5)$ & $16(23.5)$ \\
\hline Heart rate response to standing & $51(75.0)$ & $17(25)$ \\
\hline Sympathetic neuropathy & $64(94.1)$ & $4(5.1)$ \\
\hline Diastolic blood pressure response to isometric exercise &
\end{tabular}

The complete demographic data for all sixty eight patients are shown in table three. The total enrolled was sixty eight patients (all were type 2 diabetes mellitus except three) of them thirty five men and thirty three women. Variables associated with autonomic neuropathy with statistical significance were gastroparesis, nocturnal diarrhea, constipation, areas of symmetrical anhydrosis, gustatory sweating, dry skin, erectile dysfunction for men, urine retention or incontinence, palpitation, exercise intolerance, peripheral neuropathy, Insulin use, duration of diabetes, and LDLcholesterol level. The other variables are not associated with neuropathy like gender, smoking, dizziness or syncope, BMI, hypertension, the use of hypertension medication, Hba1c level, plasma glucose, total cholesterol, triglyceride, HDLcholesterol levels.

Predictors of autonomic neuropathy identified by the multivariate analysis are shown in table four. Variables which remain statistically significantly associated with autonomic neuropathy were gastroparesis, nocturnal diarrhea, areas of symmetrical anhydrosis, gustatory sweating, dry skin, erectile dysfunction for men, palpitation, exercise intolerance and insulin use.

\section{Discussion}

Forty two point six percent of the selected patients had the CAN (manifested as two abnormalities and above. The commonest parasympathetic neuropathy abnormality was abnormal beat-to-beat heart rate variation $(\mathrm{E} / \mathrm{I})$ in $61.8 \%$ and the commonest sympathetic neuropathy abnormality was diastolic blood pressure response to isometric exercise in $25 \%$ with $19.1 \%$ had two abnormalities. This finding has been confirmed in other studies, where the most informative test to assess cardiovascular autonomic neuropathy in diabetes mellitus is the abnormal beat-to-beat heart rate variation (E/I). ${ }^{11}$ In Omdurman, Sudan, evidence suggests that the CAN is present in $70 \%$ of the diabetic patients. Those who had evidence of CAN had abnormal beat-to-beat heart rate variation (E/I) in 90\%, abnormal response of heart rate to standing was positive in $81 \%$ of diabetic patients and abnormal heart rate response to Valsalva was found in $45 \%$ of the same studied patients. ${ }^{12}$

In one study conducted in India, the CAN was found in $54 \%$ of diabetic patients. About $28 \%$ had two abnormal cardiovascular reflexes. ${ }^{13}$ Abnormal beat-to-beat heart rate variation (E/I) ratio was the most sensitive test to determine autonomic neuropathy. It was abnormal in $32 \%$ patients, this was followed by abnormal heart rate response to standing, which was abnormal in $24 \%$ of the patients, Abnormal diastolic blood pressure response to isometric exercise (handgrip) test seen in $16 \%$ of the patients. Heart rate response to Valsalva maneuver ratio was abnormal in $22 \%$ of the patients. While in another study from India, the CAN was seen in $57.5 \%$, abnormal beat-to-beat heart rate variation (E/I) ratio in $30 \%$ and abnormal heart rate response to standing in $27.5 \%$. Abnormal heart rate response to Valsalva maneuver ratio in $25 \%$, abnormal diastolic blood pressure response to isometric exercise (handgrip) in $20 \%$, One 
abnormal cardiac reflex was seen in 15\%, 2 in $32.5 \%$ and 3 or more in $10 \%{ }^{14}$

Systolic blood pressure response to standing abnormality was rare in the study where it was seen only in $5.1 \%$. This result was not astonishing as the presence of postural hypotension was in the late stage in the natural history of autonomic neuropathy. ${ }^{4}$ In India, studies found the least sensitive test to detect autonomic neuropathy was postural hypotension. This was abnormal in 14-15\% of the cases. ${ }^{13,14}$

The long-term predictive power of five simple function tests with regard to all-cause mortality in individuals with diabetes from the general population found that only heart rate response to Valsalva maneuver ratio, beat-to-beat heart rate variation (E/I) ratio and diastolic blood pressure response to isometric exercise (handgrip) were predictors of survival. ${ }^{15}$

In this study, variables not associated with neuropathy were gender, smoking, dizziness or syncope, BMI, hypertension, the use of hypertension medication, HbA1c level, plasma glucose, total cholesterol, triglyceride, HDL-cholesterol levels. Several other studies have found significant relations between the CAN and peripheral neuropathy, systolic blood pressure, retinopathy and nephropathy, whereas no relationships with HbA1c, hyperlipidemia or BMI were found.16,17 Duration of diabetes in this study was not predictor of autonomic neuropathy . The EURODIAB study concluded that duration of diabetes was no longer related to incidence of the CAN when adjusted for age and sex. ${ }^{18}$

Table-3- Univariate Association of Potential Confounding Variables with Autonomic Neuropathy $(\mathrm{N}=68)$

\begin{tabular}{|c|c|c|c|c|c|}
\hline \multirow{2}{*}{\multicolumn{2}{|c|}{ Age years (Mean \pm SD) }} & Autonomic neuropathy & No autonomic neuropathy & Total & P value \\
\hline & & $53.7 \pm 9.9$ & $43.82 \pm 11.5$ & $48.0 \pm 11.9$ & 0.212 \\
\hline \multirow[t]{2}{*}{ sex } & Male N (\%) & $16(45.7)$ & $19(48.7)$ & $35(54.3)$ & \multirow[t]{2}{*}{0.598} \\
\hline & Female N (\%) & $13(39.4)$ & $20(60.6)$ & $33(48.5)$ & \\
\hline \multicolumn{2}{|c|}{ Smoking } & $10(34.5)$ & $11(28.2)$ & $21(30.9)$ & 0.825 \\
\hline \multicolumn{2}{|c|}{ Gastroparesis N (\%) } & $19(65.5)$ & $3(7.7)$ & $22(32.4)$ & $<0.0001$ \\
\hline \multicolumn{2}{|c|}{ Nocturnal diarrhea N (\%) } & $7(24.1)$ & $1(2.6)$ & $8(11.8)$ & 0.006 \\
\hline \multicolumn{2}{|c|}{ Constipation N (\%) } & $11(37.9)$ & $3(7.7)$ & $14(20.6)$ & 0.002 \\
\hline \multicolumn{2}{|c|}{$\begin{array}{l}\text { Areas of symmetrical anhydrosis, gustatory } \\
\text { sweating } N(\%)\end{array}$} & $18(62.1)$ & $3(7.7)$ & $31(30.9)$ & $<0.0001$ \\
\hline \multicolumn{2}{|c|}{ Dry skin N (\%) } & $15(51.7)$ & $3(7.7)$ & $18(26.5)$ & $<0.0001$ \\
\hline \multicolumn{2}{|c|}{ *Erectile dysfunction (\%) } & $14(87.5)$ & $2(10.2)$ & $16(45.7)$ & $<0.0001$ \\
\hline \multicolumn{2}{|c|}{ Urine retention /incontinence N (\%) } & $10(34.5)$ & $1(2.6)$ & $11(16.2)$ & $<0.0001$ \\
\hline \multicolumn{2}{|c|}{ Palpitation N (\%) } & $15(51.7)$ & $7(17.9)$ & $22(32.4)$ & 0.003 \\
\hline \multicolumn{2}{|c|}{ Dizziness/syncope N (\%) } & $25(86.2)$ & $21(53.8)$ & $46(67.6)$ & 0.005 \\
\hline \multicolumn{2}{|c|}{ *Exercise intolerance N (\%) } & $26(89.7)$ & $9(23.1)$ & $35(51.5)$ & $<0.0001$ \\
\hline \multicolumn{2}{|c|}{ BMI(Mean \pm SD) } & $26.9 \pm 6.4$ & $29.8 \pm 5.7$ & $28.6 \pm 6.1$ & 0.607 \\
\hline \multicolumn{2}{|c|}{ Peripheral neuropathy } & $29(100.0)$ & $25(64.1)$ & $54(79.4)$ & $<0.0001$ \\
\hline \multicolumn{2}{|c|}{ Insulin use $\mathrm{N}(\%)$} & $25(86.2)$ & $23(59.0)$ & $48(70.6)$ & 0.015 \\
\hline \multicolumn{2}{|c|}{ Hypertension N (\%) } & $17(43.6)$ & $16(55.2)$ & $33(48.5)$ & 0.345 \\
\hline \multicolumn{2}{|c|}{ Use anti hypertension drugs $\mathrm{N}(\%)$} & $12(41.4)$ & $10(25.6)$ & $22(32.4)$ & 0.170 \\
\hline \multicolumn{2}{|c|}{ 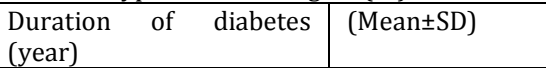 } & $13.07 \pm 7.1$ & $3.7 \pm 4.1$ & $7.74 \pm 7.2$ & 0.003 \\
\hline \multicolumn{2}{|c|}{ HbA1c(Mean \pm SD) } & $9.7 \pm 2.1$ & $10.3 \pm 2.2$ & $10.0 \pm 2.1$ & 0.900 \\
\hline \multicolumn{2}{|c|}{ Plasma glucose (Mean \pm SD) } & $218.6 \pm 86$ & $218.0 \pm 80$ & $218.2 \pm 82.1$ & 0.735 \\
\hline \multicolumn{2}{|c|}{ Total cholesterol(Mean \pm SD) } & $203.0 \pm 35.4$ & $179.1 \pm 43.3$ & $189.5 \pm 41.4$ & 0.129 \\
\hline \multicolumn{2}{|c|}{ LDL- cholesterol(Mean \pm SD) } & $124.8 \pm 24.2$ & $108.0 \pm 36.0$ & $115.3 \pm 32.2$ & 0.022 \\
\hline \multicolumn{2}{|c|}{ Triglyceride (Mean \pm SD) } & $164.4 \pm 81.4$ & $207.7 \pm 221.5$ & $188.8 \pm 174.7$ & 0.212 \\
\hline \multicolumn{2}{|c|}{ HDL-cholesterol(Mean \pm SD) } & $42.9 \pm 14.2$ & $37.6 \pm 9.0$ & $39.9 \pm 11.6$ & 0.110 \\
\hline
\end{tabular}


Table-4- Predictors of Autonomic Neuropathy Identified by Multivariate Analysis (N=68)

\begin{tabular}{|l|l|l|l|l|}
\hline Variable & $\begin{array}{l}\text { Regression } \\
\text { coefficients (B) }\end{array}$ & Standard error & Significance probability & Odds ratio \\
\hline Gastroparesis & 22.8 & 0.7 & $<0.0001$ & 3.1 \\
\hline Nocturnal diarrhea N (\%) & 12.0 & 1.1 & 0.02 & 2.4 \\
\hline Constipation & 0.13 & 0.71 & 0.05 & -1.9 \\
\hline $\begin{array}{l}\text { Areas of symmetrical anhydrosis, } \\
\text { gustatory sweating }\end{array}$ & 19.6 & 0.71 & $<0.0001$ & 2.9 \\
\hline Dry skin & 12.8 & 0.70 & $<0.0001$ & 2.5 \\
\hline *Erectile dysfunction & 17.2 & 0.8 & 2.8 \\
\hline Urine retention /incontinence & 20 & 1.0 & 0.0001 & 2.9 \\
\hline Palpitation & 4.8 & 0.5 & 0.006 & 1.5 \\
\hline Dizziness/syncope & 5.3 & 0.6 & 0.004 & 1.6 \\
\hline *Exercise intolerance & 28.8 & 0.7 & 0.007 & 3.3 \\
\hline Peripheral neuropathy & 1.8 & 10742.0 & $<0.0001$ & 21.3 \\
\hline Insulin use & 4.3 & 0.6 & 0.9 & 1.4 \\
\hline Duration of diabetes(year) & 0.018 & 0.01 & 0.02 & 0.3 \\
\hline LDL- cholesterol & 0.76 & 0.30 & $<0.0001$ & 0.01 \\
\hline *for male & & & \\
\hline
\end{tabular}

\section{Conclusion}

Various variables can predict the CAN, which affects more than $40 \%$ of diabetic patients in Basrah.

\section{References}

1. Vinik, A. I., Freeman, R. \& Erbas, T. (2003). "Diabetic Autonomic Neuropathy," Seminars in Neurology, Dec 2003; 23(4):365-72.

2. Boulton, A. J. M., Vinik, A. I., Arezzo, J. C., Bril, V., Feldman, E. L., Freeman, R., et al. (2006). "Etal."Diabetic Neuropathies: A Statement by the American Diabetes Association," Diabetes Care. 2005 Apr; 28(4):956-62.

3. Vinik, A. I., Maser, R. E., Mitchell, B. D. \& Freeman, R. (2003). "Diabetic Autonomic Neuropathy," Diabetes Care. 2003 May; 26(5):1553-79.

4. Consensus Statement: Report and Recommendations of the San Antonio Conference on Diabetic Neuropathy. (1988). American Diabetes Association
American Academy of Neurology, Diabetes Care. 1988 Jul-Aug; 11(7):592-7.

5. Vinik, A. I. \& Ziegler, D. (2007). "Diabetic Cardiovascular Autonomic Neuropathy," Circulation. 2007 Jan; 115(3):387-97.

6. Pfeifer, M. A., Weinberg, C. R., Cook, D. L., Reenan, A., Halter, J. B., Ensinck, J. W. \& Porte, D. (1984). "Autonomic Neural Dysfunction in Recently Diagnosed Diabetic Subjects," Diabetes Care. 1984 Sep-Oct; 7(5):447-53.

7. Mackay, J. D., Page, M. M., Cambridge, J. \& Watkins, P. J. (1980). "Diabetic Autonomic Neuropathy: The Diagnostic Value of Heart Rate Monitoring," Diabetologia. 1980 Jun; 18(6):471-8.

8. Kahn, R. (1992). "Proceedings of a Consensus Development Conference on Standardized Measures in Diabetic Neuropathy," Diabetes Care .1992 15(Suppl. 3):1080-107.

9. Young, M. J., Boulton, A. J. M., Macleod, A. F., Williams, D. R. R. \& Sonksen, P. H. (1993). "A Multicenter Study of the Prevalence of Diabetic Peripheral 
Neuropathy in the United Kingdom Hospital Clinic Population," Diabetologia. 1993 Feb; 36(2):150-4.

10. Ziegler, D., Dannehl, K., Muhlen, H., Spuler, M. \& Gries, F. A. (1992). "Prevalence of Cardiovascular Autonomic Dysfunction Assessed by Spectral Analysis, Vector Analysis, and Standard Tests of Heart Rate Variation and Blood Pressure Responses at Various Stages of Diabetic Neuropathy," Diabetic Medicine. 1992 Nov; 9(9):806-14.

11. May, O. \& Arildsen, H. (2000). "Assessing Cardiovascular Autonomic Neuropathy in Diabetes Mellitus: How Many Tests to Use?," Journal of Diabetes and its Complications. 2000 Jan-Feb; 14(1):7-12.

12. Hussein, A., Fatah, T. A., Sidig, A., Hamad, A., Gadour, M. O., Yassien, F., Ali, A. B. M., Saad, A. \& Aldar, M. M. (2011). "Frequency and Clinical Pattern of Autonomic Neuropathy in Adult Diabetic Sudanese Patients," International Journal of the Physical Sciences. 2011 Jan; 6(2): 308-312.

13. Basu, A. K., Bandyopadhyay, R., Chakrabarti, S., Paul, R. \& Santra, S. (2010). "A Study on the Prevalence of Cardiac Autonomic Neuropathy in Type2 Diabetes in Eastern India," Journal, Indian Academy of Clinical Medicine. 2010; 11(3): 190-4.

14. Mehta, S., Mathur, D., Chaturvedi, M. \& Verma, K. (2002). "Incidence of Cardiac
Autonomic Neuropathy and Its Correlation with Retinopathy, MicroAlbuminuria and Glycated Haemoglobin in Non-Insulin Dependent Diabetes Mellitus," Journal of the Indian Medical Association. 2002; 100:141-3.

15. May, 0. \& Arildsen, H. (2011). "LongTerm Predictive Power of Simple Function Tests for Cardiovascular Autonomic Neuropathy in Diabetes: A Population-Based Study," Acta Diabetologica. 2011 Dec; 48(4):311-6.

16. Chen, H.- T., Lin, H.- D., Won, J. G. S., Lee, C.- H., Wu, S.- C., Lin, J.- D., Juan, L.- Y., Ho, L.- T. \& Tang, K.- T. (2008). "Cardiovascular Autonomic Neuropathy, Autonomic Symptoms and Diabetic Complications in 674 Type 2 Diabetes," Diabetes Research and Clinical Practice. 2008 Nov; 82(2):282-90.

17. Lacigová, S., Safránek, P., Cechurová, D., Krcma, M., Vísek, J., Jankovec, Z., Zourek, M., Haladová, I. \& Rusavý, Z. (2007). "Could We Predict Asymptomatic Cardiovascular Autonomic Neuropathy in Type 1 Diabetic Patients Attending Out-Patients Clinics?," Wiener Klinische Wochenschrift. 2007; 119(9-10):303-8.

18. Witte, D. R., Tesfaye, S., Chaturvedi, N., Eaton, S. E. M., Kempler, P. \& Fuller, J. H. (2005). "Risk Factors for Cardiac Autonomic Neuropathy in Type 1 Diabets Mellitus," Diabetologia. 2005 Jan; 48(1):164-71. 